\title{
A Diagnostic Criterion for Power Capacitor in Distribution Network
}

\author{
Xie Feng, Jiang Daozhuo, Du Yi, Chen Ke, Chen Feng \\ Zhejiang University, Hangzhou, China \\ 1007504844@qq.com
}

Keywords: power capacitor; voltage compensation; diagnostic criterion; simulation

\begin{abstract}
In power system, voltage quality has always been an important indicator to evaluate the reliability and stability of power supply. In engineering, to solve the problem of voltage drop at the end of power line, power capacitors are often used to maintain voltage stability. Taking a reactive compensation capacitor as an example, this paper analyzes the internal structure of this capacitor and provides a mathematical modeling. And on this basis, a diagnostic criterion is proposed aiming at the internal fault of capacitor and its reliability and feasibility has been verified through a series of simulation and analysis.
\end{abstract}

\section{Introduction}

In power grid construction, voltage quality has always been an important indicator to value whether the quality of power supply meets certain criterion. Comparing to transmission network, the construction of distribution network in China is seriously lagged behind with its weak grid, aging facilities, small diameter wirings and huge numbers of distribution transformers ${ }^{[1]}$. Many lines of the distribution network have multiples of large capacity motors or quickly changing industrial loads and it has caused many serious problems.

The structure and control of series compensation device is relatively simple. Not only does it have the function of improving the static-stability limit and regulating the distribution of power flow, but also has a characteristic that the load will "spontaneously adapt" to the voltage regulation and realtime response ${ }^{[2-4]}$, which by contrast, having a better prospect for economy and development.

Once the high voltage capacitors in D-FSC (distribution-fixed series capacitor) are damaged because of the aging capacitor components, it will affect the safe operation of power grid $^{[5]}$. At present, the power capacitor device generally uses some conventional safety operation protection measures such as current (or voltage) differential protection proposed by [6]. A diagnostic criterion presented by [7] values the damage condition by calculating the capacitance through detecting the voltage and current directly on both sides of the capacitor. This criterion can use the value of system detection directly and reduce the additional equipment, however, it ignores the harmonics in power system which resulting in a low precision.

This paper analyses the basic principles of D-FSC and through modeling and mathematical reasoning of the power capacitor, this paper puts forward a criterion method to judge the internal faults of capacitor when system exists harmonics. Finally, the paper verifies the effectiveness and feasibility of the criterion through simulation experiment.

\section{The internal structure of capacitor}

The D-FSC device is installed in series between two tapping points of the distribution line (connecting points of distribution transformer branch and main line). The structure has been shown in Fig. 2. 


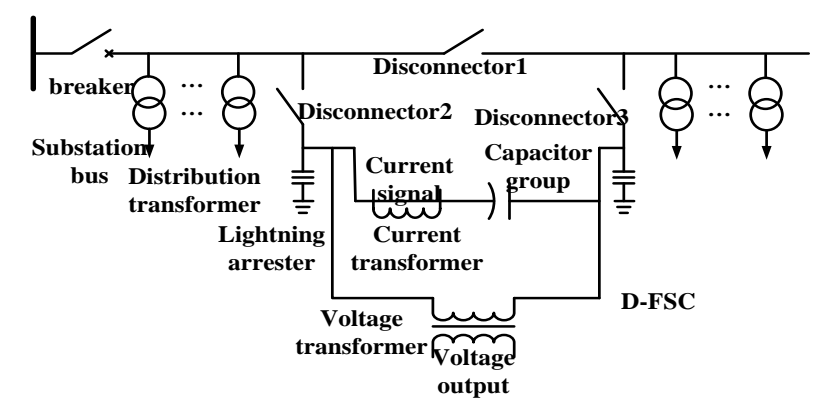

Figure 1. $\quad$ Structure chart of D-FSC.

The internal structure of series compensation capacitor has been shown in Fig. 3. The capacitors in the device are paralleled by multiples of internal small capacitors and resistances, where each small capacitor is connected in series with an internal fuse.

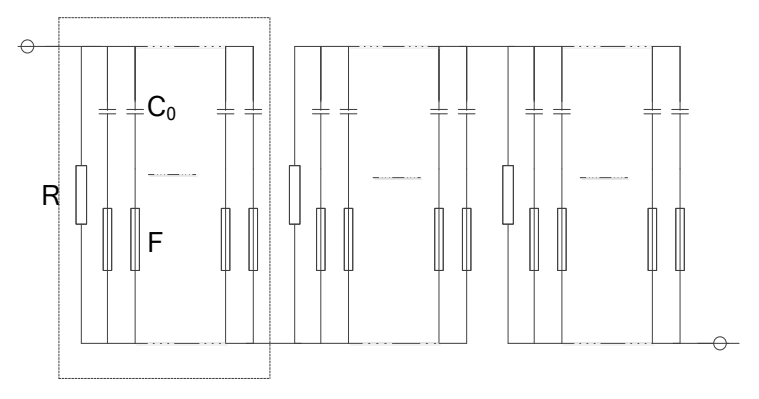

Figure $2 . \quad$ Internal structure of the capacitor.

\section{Analasis of capacitor modeling}

\section{Modeling of Power Capacitor}

Large capacity power capacitor is generally composed of dozens or even hundreds of small capacitors in series or paralleled forms. This paper uses capacitor BAM11/ $\sqrt{3}-334-1 \mathrm{~W}$ as a case to analyze, which is mainly composed by 36 capacitors with 12 in parallel and 6 in series connection. The value of paralleled resistance is very large, and the loss of capacitor's active power is very small, which means the resistance could be negligible. The capacitance is $4 \mathrm{C}_{0}=26.3592 \mathrm{uF}$, and the simplified model is shown in Fig. 4. Then the value of capacitance $\mathrm{C}$ can be calculated by the following equation:

$$
C=\frac{I}{\omega U} \text {. }
$$

In this equation: $I$ - the effective value of the current flowing through the capacitor, $U$ - the effective value of the capacitor voltage, $\omega$-the angular frequency.

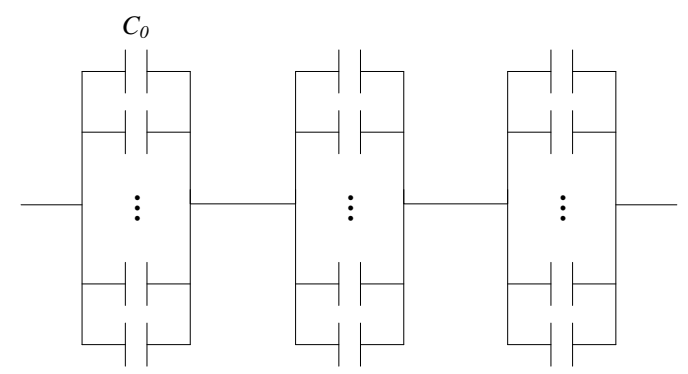

Figure $3 . \quad$ Simplified internal structure of the capacitor.

After analyzing the capacitor, value of this capacitor when different damage occurred within the small capacitors can be calculated by the following: 


$$
C=\frac{1}{\frac{1}{x C_{0}}+\frac{1}{y C_{0}}+\frac{1}{z C_{0}}}=\frac{x y z C_{0}}{x y+y z+x z} .
$$

In the equation: $\mathrm{C}_{0}$ —value of every small internal capacitor, $\mathrm{x}, \mathrm{y}, \mathrm{z}$ - the number of capacitors that can work regularly in each parallel group of the three.

Therefore, the relative variation percentage of the capacitance can be expressed as:

There are two kinds of circumstances as following:

$$
\frac{\Delta C}{C}=\left|\frac{C-4 C_{0}}{4 C_{0}}\right|=1-\frac{1}{4(1 / x+1 / y+1 / z)} .
$$

(1)Two small capacitors are damaged separately in two parallel groups. That is, $\mathrm{x}, \mathrm{y}, \mathrm{z}$ in one of 12 , and the remaining two are 11 , such as $(x, y, z)=(11,11,12)$, then

$$
\frac{\Delta \mathrm{C}}{\mathrm{C}}=1-\frac{1}{4\left(\frac{1}{11}+\frac{1}{11}+\frac{1}{12}\right)}=5.7143 \% \text {. }
$$

(2)Two small capacitors are damaged in the same parallel group. That is $\mathrm{x}, \mathrm{y}, \mathrm{z}$ in one of 10 , and the other two are 12 , such as $(x, y, z)=(10,12,12)$, then

$$
\frac{\Delta \mathrm{C}}{\mathrm{C}}=1-\frac{1}{4\left(\frac{1}{10}+\frac{1}{12}+\frac{1}{12}\right)}=6.2500 \% \text {. }
$$

Therefore, when two small capacitors inside are damaged, the variation percentage of capacitance can be approximated as $5.9822 \%$ by taking the average of the two results above. By the same token, we can calculate the relative variation percentage of the capacitance when there is 0,1 , or 3 small capacitors damaged. The results are listed as Table 1.

TABLE I. The VARIATION PERCENTAGE OF CAPACITANCE WITH INTERNAL FAUlTS IN CAPACITOR

\begin{tabular}{cc}
\hline The Damaged Number & $\Delta \mathrm{C} / \mathrm{C} \%$ \\
\hline 0 & 0 \\
1 & 2.9412 \\
2 & 5.9822 \\
3 & 9.0577 \\
\hline
\end{tabular}

By monitoring the capacitor current and voltage and using (4) to obtain the real-time capacitance, and then according (5) and (6), we can calculate the variation of capacitance to diagnose the faults of capacitor. Criterion table can be obtained as follows:

\begin{tabular}{cc} 
TABLE II. & CRITERION TABLE \\
\hline $\begin{array}{c}\text { The Damaged number of } \\
\text { small capacitors }\end{array}$ & $\Delta C / C(\%)$ \\
\hline 0 & $(0,1.4706)$ \\
2 & $(1.4706,4.4617)$ \\
$\geq 3$ & $(4.4617,7.5200)$ \\
\hline
\end{tabular}

\section{Improved Criterion to Improve Accuracy}

In general, harmonics always exists in power system. Using (4) to calculate capacitance ignores this situation and possibly cause error diagnosis such as follows:

The capacitor is intact and its capacitance $4 \mathrm{C}_{0}$ is $26.3592 \mathrm{uF}$. The effective value of fundamental voltage through the capacitor is $1 \mathrm{kV}$. Assuming there exists harmonics in system and the 3, 5, 7, 11 harmonic amplitude value is $10 \%, 5 \%, 3 \%$ and $2 \%$ respectively. By detecting effective value of capacitor voltage $U$ and effective value of capacitor current $I$ and calculating capacitance $C=29.266 u F$ by (4), the relative variation percentage of capacitance is $9.932 \%$. Thus make a judgment that there are 3 damaged capacitors and it exists error diagnosis. Then operating personnel will cut the D-FSC according to original criterion and cause unnecessary waste of time and resources. 
Therefore, considering the situation of harmonic characteristics, Using FFT expansion to detected voltage and current and we can get the fundamental wave and harmonics of the voltage and current is $\mathrm{U}_{(1)}, \mathrm{U}_{(3)}, \mathrm{U}_{(5)} \ldots \mathrm{I}_{(1)}, \mathrm{I}_{(3)}, \mathrm{I}_{(5)}$...Therefore, the capacitance of each harmonic $\mathrm{C}_{(i)}$ is

$$
C_{(i)}=\frac{I_{(i)}}{i \omega U_{(i)}} .
$$

In the equation: $i$ - the times of harmonics.

In theory, the calculated capacitance value of the fundamental and other harmonics are the same. However, there are differences in practice. So, according to the proportion of the fundamental and the harmonics, the estimation of capacitance can be estimated by (10):

$$
C_{\text {detect }}=\frac{\sum_{i} I_{(i)} C_{(i)}}{\sum_{i} I_{(i)}} .
$$

After estimating the capacitor, get formula (11).

$$
\Delta C=\frac{C_{\text {detect }}-C}{C} .
$$

According to the variation percentage of the correcting capacitance by (10) and (11), and with table 2, we can detect the faults in capacitor.

\section{Simulation and analysis}

From the construction of capacitor model, it can be found that the results from conventional criterion and improved criterion are the same when there is no harmonics (improved criterion is equivalent to the fundamental component only when there are no harmonics). However, the presence of harmonics in system will get different results. We will verify the new criterion from the simulation below.

\section{Internal Faults Simulation}

When the system exists harmonics and the capacitor groups are not destructed, the harmonic contents of capacitor's voltage and current is show in Fig. 5 and Fig. 6:

Its effective value of the fundamental voltage $\mathrm{U}_{(1)}$ is $240.667 \mathrm{~V}$; and the effective value of fundamental current $I_{(1)}$ is $1.9912 \mathrm{~A}$; the five harmonic $U_{(5)}$ is $10.329 \mathrm{~V}$; $\mathrm{I}_{(5)}$ is $0.4381 \mathrm{~A}$; the seven harmonic $\mathrm{U}_{(7)}$ is $4.616 \mathrm{~V}$; $\mathrm{I}(7)$ is $0.2551 \mathrm{~A}$; Capacitance can be obtained from the conventional criterion $C=27.1469 \mathrm{uF}$ and the variation percentage of capacitance is $2.902 \%$; Capacitance from improved criterion $C=26.3802 \mathrm{uF}$ and the variation percentage of capacitance is $0.08 \%$. According to the criterion table, in the conventional criterion, because of the influence of harmonics, there exists misjudgment that 1 capacitor is damaged while the improved criterion won't be influenced by the harmonics.

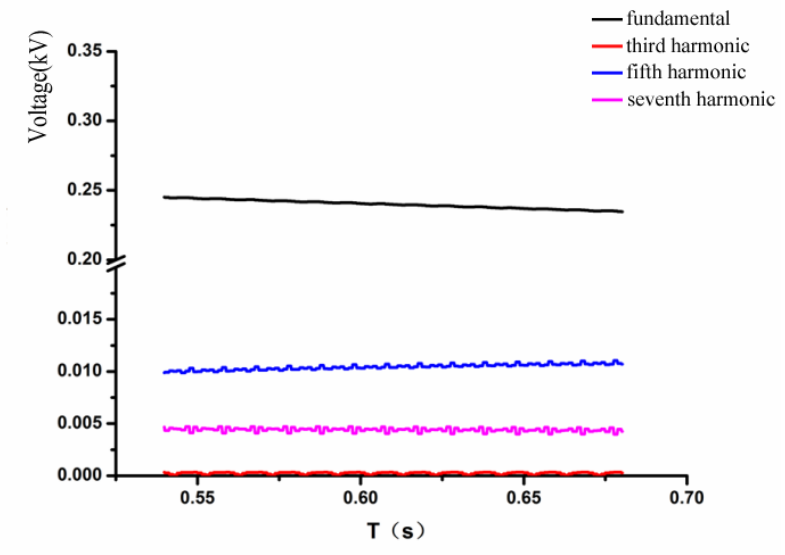

Figure 4. The voltage of D-FSC without internal destruction. 


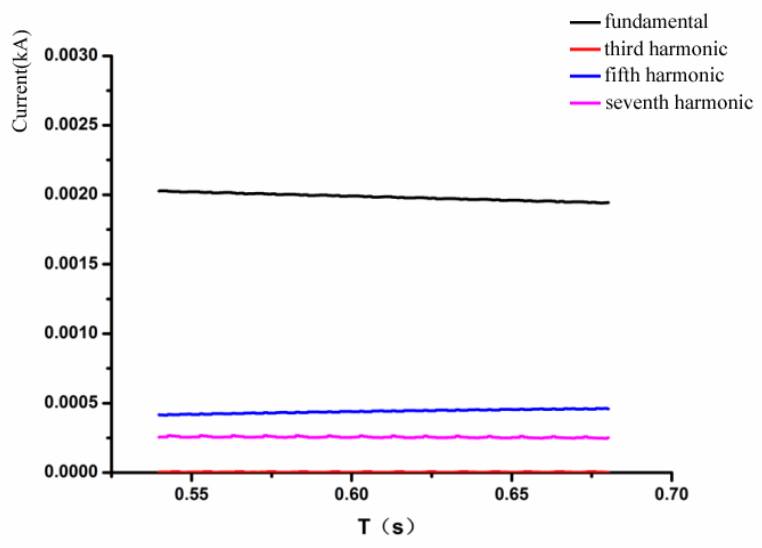

Figure 5. The current of D-FSC without internal destruction.

When two small capacitors are damaged in the same in the same parallel group and other capacitors work regularly, the harmonic contents of capacitor voltage and current are shown in Fig. 7 and Fig. 8.

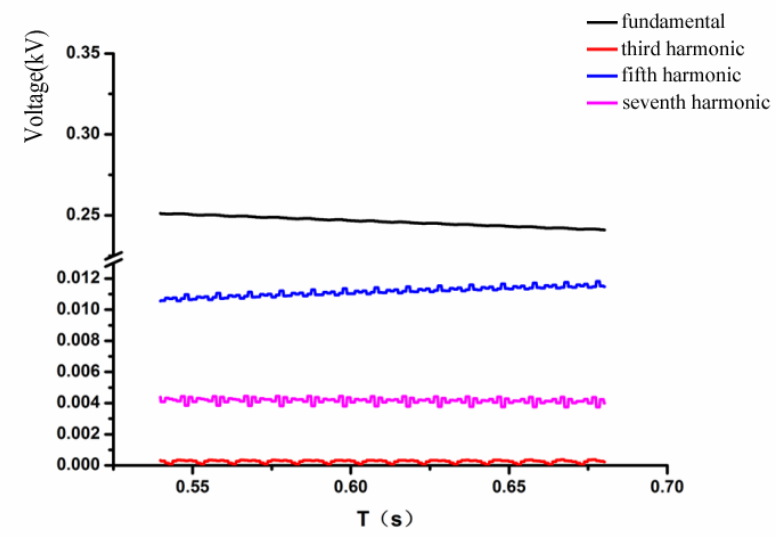

Figure 6. The voltage of D-FSC with internal destruction.

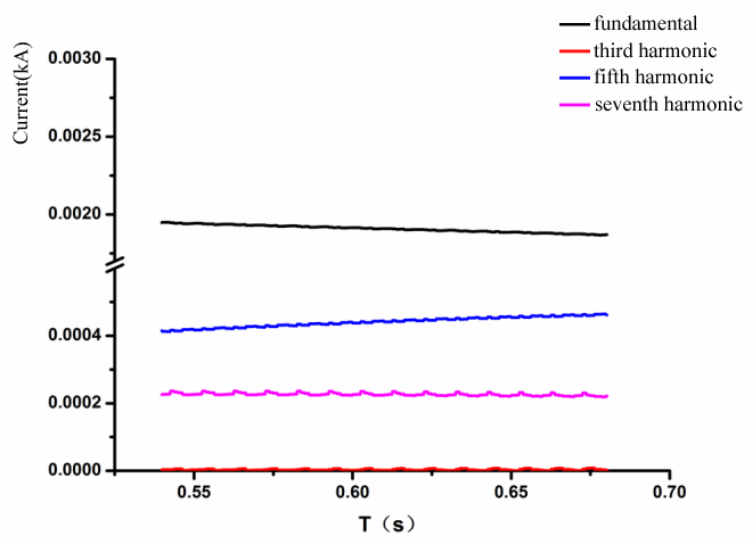

Figure 7. The current of D-FSC with internal destruction.

Its effective value of fundamental voltage $\mathrm{U}_{(1)}$ is $246.724 \mathrm{~V}$; The effective value of fundamental current $\mathrm{I}_{(1)}$ is $1.915 \mathrm{~A}$; five times harmonic $\mathrm{U}_{(5)}$ is $11.030 \mathrm{~V}$; $\mathrm{I}_{(5)}$ is $0.438 \mathrm{~A}$; seven times harmonic $\mathrm{U}(7)$ is $4.065 \mathrm{~V}$; $\mathrm{I}_{(7)}$ is $0.226 \mathrm{~A}$; From conventional criterion, capacitance $C$ is $25.487 \mathrm{uF}$ and the variation percentage of capacitance is $3.421 \%$; Misjudge again as this criterion only found 1 capacitor failure. From improved criterion, we get capacitance $C$ is $24.855 \mathrm{uF}$ and the variation percentage of capacitance is $6.052 \%$. There are 2 capacitors in failure which is a correct judgment.

Comparison of Improved Criterion

Seeing from Table 3, the improved criterion is more ideal as in the conventional criterion, misjudge happens when there is no capacitor in destruction and when there exists internal fault, it 
will consider the small capacitors inside normal. So the conventional criterion loses accuracy when system has harmonics and the improved criterion just solves this problem. It can accurately judge whether the fault occurs even considering the harmonics and the damaged number of capacitors.

TABLE III. CAPARISON OF CRITERIONS

\begin{tabular}{|c|c|c|c|}
\hline Method & $\begin{array}{c}\text { The actual damaged } \\
\text { situation }\end{array}$ & $\Delta C / C(\%)$ & $\begin{array}{l}\text { Judgment of the fault } \\
\text { capacitors }\end{array}$ \\
\hline \multirow{4}{*}{ Conventional criterion } & 0 & 2.902 & 1 \\
\hline & 1 & 0.073 & 0 \\
\hline & 2 & 3.421 & 1 \\
\hline & 3 & 7.697 & 3 \\
\hline \multirow{4}{*}{ Improved criterion } & 0 & 0.080 & 0 \\
\hline & 1 & 2.436 & 1 \\
\hline & 2 & 6.052 & 2 \\
\hline & 3 & 10.458 & 3 \\
\hline
\end{tabular}

\section{Conclusion}

This paper analyzes the basic principles of how the D-FSC improving the voltage characteristics in the radial line and draws a conclusion that the series compensation device can "spontaneously adapt" to the voltage regulation. And based on inducting the original diagnostic method of D-FSC capacitor, it presents a new diagnostic criterion to detect the internal fault of capacitors when system is influenced by harmonics. By comparing to the original criterion through simulation and analyzing the diagnostic criterion of D-FSC internal fault, the new criterion can effectively solve the influence of harmonics and accurately judge the internal fault of capacitor. It is more superiority comparing to the original criterion.

\section{References}

[1] Dai Xiaoliang. Application of Reactive Compensation Technology for Distribution System[J]. Power System Technology.1999, 23(6): 1-7.

[2] Zhuo Guying, Jiang Daozhuo, LIANG Yiqiao. A Research of D-FSC for Improving Voltage Quality in Distribution Networks[J]. Power System Protection and Control. 2013, 8: Vol. 41.

[3] J.M.Magowan, Voltage Performance of Series Capacitors in Transmission and Distribution Lines[J]. The Institution of Electrical Engineers, paper 2252S. 1957: 505-516.

[4] Alberto D. Del Rosso, Claudio A. Cañizares, Victor M. Doña.A Study of TCSC Controller Design for PowerSystem Stability Improvement [J]. IEEE Transactions on Power Systems.2003, 18(4): 1487-1496.

[5] S. A. Miske. Considerations for the Application of SeriesCapacitors to Radial Power Distribution Circuits. IEEE Transactions on Power Delivery, 2001, 16(2).

[6] Larry Morgan. James M. Barcus. Satoru Ihara. Distribution Series Capacitor with High-energy VaristorProtection[J]. IEEE Transactions on Power Delivery. 1993,8(3): 1413-1419.

[7] IEEE Std C37.99-2000 IEEE Guide for the Protection of Shunt Capacitor Banks[S]. New York, NY, USA: The Institute of Electrical and Electronics Engineers, 2000.

[8] QIAO Lei. Estimated Means of Reactive Power for Distributed Small Hydro Stations Based on 0ptimal Power Flow [J]. Electric Power System and Its Automation Journal 2008, 20(2): 88-93. 